\title{
Surfactant mediated growth of Ti/Ni multilayers
}

\author{
Mukul Gupta, ${ }^{1, a)}$ S. M. Amir, ${ }^{1}$ Ajay Gupta, ${ }^{1}$ and Jochen Stahn ${ }^{2}$ \\ ${ }^{1}$ UGC-DAE Consortium for Scientific Research, University Campus, Khandwa Road, Indore 452001, India \\ ${ }^{2}$ Laboratory for Neutron Scattering, Laboratory for Neutron Scattering, Paul Scherrer Institut, \\ CH-5232 Villigen PSI, Switzerland
}

(Received 18 January 2011; accepted 21 February 2011; published online 11 March 2011)

\begin{abstract}
The surfactant mediated growth of Ti/Ni multilayers is studied. They were prepared using ion beam sputtering at different adatom energies. It was found that the interface roughness decreased significantly when the multilayers were sputtered with Ag as surfactant at an ion energy of $0.75 \mathrm{keV}$. On the other hand, when the ion energy was increased to $1 \mathrm{keV}$, it resulted in enhanced intermixing at the interfaces and no appreciable effect of Ag surfactant could be observed. On the basis of the obtained results, the influence of adatom energy on the surfactant mediated growth mechanism is discussed. (C) 2011 American Institute of Physics. [doi:10.1063/1.3565161]
\end{abstract}

$\mathrm{Ti} / \mathrm{Ni}$ multilayers have found applications in many emerging areas including neutron mirrors, ${ }^{1-3}$ soft $\mathrm{x}$-ray optics, ${ }^{4-6}$ and shape memory alloys for making microelectronic mechanical systems devices. Also, this system is considered as a model system for demonstrating the solid state amorphisation reaction. ${ }^{8-10}$ Therefore, the Ti/Ni multilayer system is interesting both, from the basic as well as from the application point of view. In particular, the large difference between the neutron scattering length densities (SLDs) of Ti $\left(-1.95 \times 10^{-6} \AA^{-2}\right)$ and $\mathrm{Ni}\left(9.41 \times 10^{-6} \AA^{-2}\right)$ makes Ti/Ni multilayers an ideal candidate in neutron optics. Attempts have been made to further optimize this multilayer system either by increasing the contrast by adding hydrogen ${ }^{1,11}$ or by adding interdiffusion barriers such as $\mathrm{Cr}$ and $\mathrm{C}^{6,12,13}$

The surface free energy $(\gamma)$ for the average face of $\mathrm{Ni}$, $\mathrm{Ti}$, and $\mathrm{Ag}$ are $\gamma_{\mathrm{Ni}}=2.4 \mathrm{~J} \mathrm{~m}^{-2}, \gamma_{\mathrm{Ti}}=2.1 \mathrm{~J} \mathrm{~m}^{-2}$, and $\gamma_{\mathrm{Ag}}$ $=1.2 \mathrm{~J} \mathrm{~m}^{-2}$, respectively. ${ }^{14,15}$ A difference in the $\gamma$ is known to produce multilayers with asymmetric interface as wetting of the material with a lower $\gamma$ will take place on a material with higher $\gamma$. In the opposite case agglomeration due to dewetting takes place. Therefore, from the difference in the $\gamma$, it is expected that the Ti-on-Ni interface should be smoother than the Ni-on-Ti interface in a Ti/Ni multilayer. This asymmetry may result in increased roughness and stress as the growth of the multilayer takes place. Such an increase in roughness and stress leads to a decrease in the peak reflectivity and to an enhanced interdiffusion. In a neutron monochromator or supermirror several hundreds of repetitions are required for the optimum performance of the device. In this situation the stress can buildup so intensely that the deposited film may peel-off from the substrate as observed experimentally. ${ }^{16}$

The addition of a surface active species (a surfactant) might balance the asymmetry of the interfaces. ${ }^{17}$ A surfactant essentially has a significantly lower $\gamma$ than the multilayer components so it leads to a wetting and thus to smoother interfaces. If in addition it is not incorporated in either of the compounds, it will flow on top during the deposition and thus allow for a layer-by-layer type growth throughout the complete film. ${ }^{18}$ In the case of Ti/Ni multilayers, no attempt has been made yet to study the surfactant mediated growth,

${ }^{a)}$ Electronic mail: mgupta@csr.res.in. although surfactants have been used in other types of multilayers, e.g., $\mathrm{Sb}$ as surfactant in $\mathrm{Si} / \mathrm{Ge}$ multilayers, ${ }^{19} \mathrm{O}$ and $\mathrm{Ag}$ as surfactants in $\mathrm{Cu} / \mathrm{Co}$ multilayers, ${ }^{20,21}$ etc. In addition, the role of adatom energy on the surfactant mechanism has not been studied.

In this letter, we present the results of surfactant mediated growth of Ti/Ni multilayers, prepared using ion beam sputtering (IBS) at incident ion energy $E_{\mathrm{Ar}^{+}}=0.75 \mathrm{keV}$ and $1.00 \mathrm{keV}$. Two otherwise identical sets of samples have been prepared on silicon (100) substrates at room temperature with following deposition sequences:

$$
\begin{aligned}
& \text { A: } \operatorname{Ti}(10 \mathrm{~nm}) /[\operatorname{Ti}(6 \mathrm{~nm}) / \operatorname{Ni}(4 \mathrm{~nm})]_{10}, \\
& \text { B:Ti(10 nm)/Ag(0.2 nm) } /[\operatorname{Ti}(6 \mathrm{~nm}) / \mathrm{Ni}(4 \mathrm{~nm})]_{10}, \\
& \text { C:Ti(10 nm)/[Ti(6 nm)/Ag(0.2 nm)/Ni(4 nm) }]_{10} .
\end{aligned}
$$

The $\operatorname{Ti}(10 \mathrm{~nm})$ layer deposited on the substrate is a buffer layer to minimize the substrate effects. The ion beam produced with a rf-ion source (Veeco) was kept incident at angle of $45^{\circ}$ with respect to the target. The base pressure was less than $5 \times 10^{-8}$ mbar prior to the deposition while the pressure during the deposition was $5 \times 10^{-4}$ mbar due to the flow of the Ar gas (purity 99.9995\%) in the ion source and rf-neutralizer. Each target was presputtered for about $15 \mathrm{~min}$ to remove contaminations from the target. The presputtering of $\mathrm{Ti}$ also helped to minimize the oxygen partial pressure. The targets mounted on a rotary motion were alternatively rotated to deposit the multilayer structures. The deposition rates were $2.3 \mathrm{~nm} / \mathrm{min}$ and $1.66 \mathrm{~nm} / \mathrm{min}$ for $\mathrm{Ni}$ and Ti, respectively for $E_{\mathrm{Ar}^{+}}=0.75 \mathrm{keV}$ and $2.52 \mathrm{~nm} / \mathrm{min}$ and $2.23 \mathrm{~nm} / \mathrm{min}$ for $\mathrm{Ni}$ and $\mathrm{Ti}$, respectively for $E_{\mathrm{Ar}^{+}}$ $=1.00 \mathrm{keV}$.

The preliminary characterization of the samples were carried out using x-ray $(\mathrm{Cu} K \alpha)$ reflectivity (XRR). X-ray diffraction (XRD) measurements were carried out to measure the structure of the deposited samples. Figure 1 shows the XRD pattern of the samples A. The peaks observed correspond to $\mathrm{Ni}(111)$, Ti(002), and $\mathrm{Ti}(101)$. As can be seen, the peaks are relatively broader for the sample prepared using a lower $\mathrm{Ar}^{+}$ion energy. The average grain size can be calculated using the Scherrer formula: $t=0.9 \lambda / \Delta \theta \cos \theta$, where $t$ is the grain size, $\Delta \theta$ is an angular width of the reflection, $\theta$ is the Bragg angle, and $\lambda$ is the wavelength of the radiation used. The grain size of the sample prepared using $E_{\mathrm{Ar}^{+}}$ 


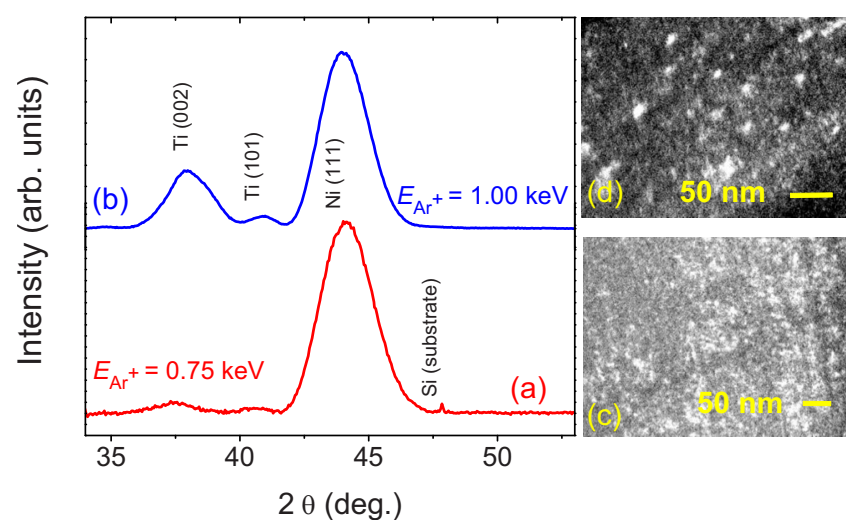

FIG. 1. (Color online) $\mathrm{X}$-ray $(\mathrm{Cu} K \alpha)$ diffraction pattern and TEM dark field images of samples prepared with $E_{\mathrm{Ar}^{+}}=0.75 \mathrm{keV}$ (a) and (c); with $E_{\mathrm{Ar}^{+}}=1.00 \mathrm{keV}(\mathrm{b})$ and (d), respectively.

$=1.00 \mathrm{keV}$ was $4.8 \pm 0.1 \mathrm{~nm}$ which is somewhat larger as compared to $4.2 \pm 0.1 \mathrm{~nm}$ for the sample with $E_{\mathrm{Ar}^{+}}$ $=0.75 \mathrm{keV}$. All XRD patterns of samples with varying surfactant contents but same incident ion energy were identical. In order to corroborate the XRD results, transmission electron microcopy (TEM) measurements were carried out. Figures 1(c) and 1(d) show TEM dark field images of samples prepared using incident ion energy of $0.75 \mathrm{keV}$ and $1.00 \mathrm{keV}$, respectively. The bright spots in the images correspond to $\mathrm{Ni}$ particles. The average grain size calculated using TEM images matches well with those obtained using XRD measurements.

Neutron reflectivity (NR) measurements were carried out to measure the depth profile of the SLDs. The samples prepared with $E_{\mathrm{Ar}^{+}}=0.75 \mathrm{keV}$ were measured at the reflectometer apparatus for multi optional reflectometry at SINQ/PSI, Switzerland in the time of flight mode, ${ }^{22}$ and the ones with $E_{\mathrm{Ar}^{+}}=1.00 \mathrm{keV}$ in the monochromatic mode $(\lambda=4.4 \AA)$ at SuperADAM instrument at Institut Laue-Langevin (ILL), France. The resulting curves are shown in Fig. 2. They were fitted using Parratt's formulism. ${ }^{23}$ While the NR pattern for samples $\mathrm{A}$ and B with $E_{\mathrm{Ar}^{+}}=0.75 \mathrm{keV}$ can be fitted well assuming their nominal structures, the one for sample $\mathrm{C}$ shows a broad hump riding on top of total thickness oscillations. This hump could be fitted assuming a layer of about 2

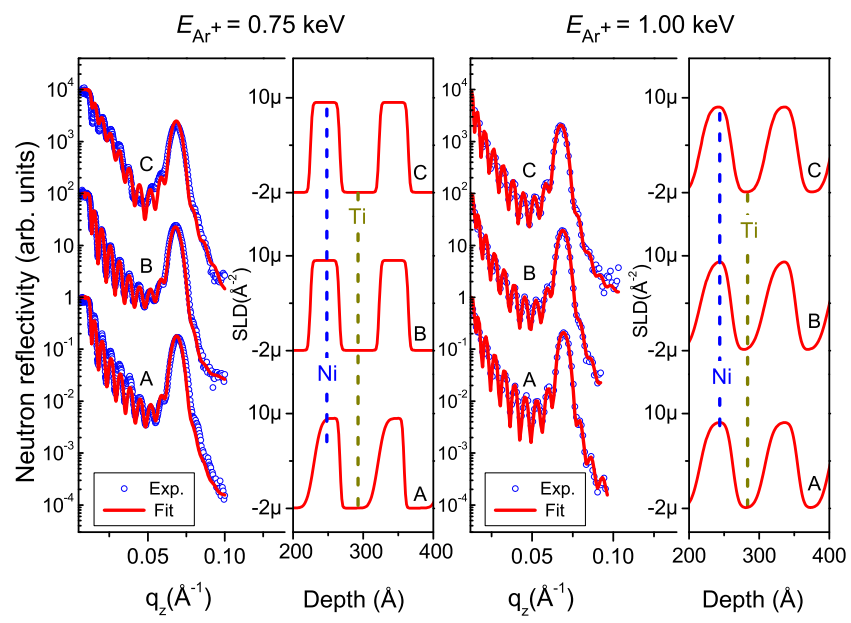

FIG. 2. (Color online) NR and SLD profiles of the Ti/Ni multilayers (samples A, B, and C) prepared with $E_{\mathrm{Ar}^{+}}=0.75 \mathrm{keV}$, and with $E_{\mathrm{Ar}^{+}}$ $=1.00 \mathrm{keV}$. The reflectivities are scaled for clarity.
TABLE I. Fitted NR parameters (reflectivity $\mathcal{R}$ and rms roughness $\sigma$ ) for Ti/Ni multilayers.

\begin{tabular}{|c|c|c|c|c|c|c|}
\hline \multirow{2}{*}{$\frac{\mathrm{Ar}^{+} \text {ion energy }}{\text { Samples }}$} & \multicolumn{3}{|c|}{$0.75 \mathrm{keV}$} & \multicolumn{3}{|c|}{$1 \mathrm{keV}$} \\
\hline & A & B & $\mathrm{C}$ & $\mathrm{A}$ & B & C \\
\hline $\mathcal{R}( \pm 1 \%)$ & 18.6 & 24.4 & 23.7 & 16.3 & 17.4 & 18.1 \\
\hline$\sigma_{[\text {Ni-on-Ti }]}( \pm 0.05 \mathrm{~nm})$ & 0.8 & 0.2 & 0.2 & 1.20 & 1.03 & 0.90 \\
\hline$\sigma_{[\text {Ti-on-Ni] }}( \pm 0.05 \mathrm{~nm})$ & 0.2 & 0.2 & 0.2 & 0.64 & 0.64 & 0.60 \\
\hline
\end{tabular}

$\mathrm{nm}$ on top of the multilayer structure with a SLD close to that of bulk Ag. It looks like all the Ag from all Ti-Ni interfaces accumulated on top of the film. The details of fitting are given in Table I. As can be seen there, the reflected intensity $(\mathcal{R})$ at the Bragg peak $\left(q_{z}=0.068 \AA^{-1}\right)$ for the sample prepared at $0.75 \mathrm{keV}$ without any surfactant was $18.6 \%$ while it increases to $24.4 \%$ when $\mathrm{Ag}$ was added at the buffer Ti layer, and $23.7 \%$ when the surfactant was introduced at each Ti layer. Whereas, in case of the $1 \mathrm{keV}$ sample $\mathcal{R}$ only increases marginally with addition of $\mathrm{Ag}$.

The SLD depth profiles for samples A, B, and C obtained by fitting of the NR data, are also shown in Fig. 2 exemplarily for the depth between $200-400 \AA$. The SLD depth profile for the reference sample A deposited at $E_{\mathrm{Ar}^{+}}$ $=0.75 \mathrm{keV}$, shows an asymmetry with an abrupt Ti-on-Ni and a skewed Ni-on-Ti interface. As expected, Ti with lower $\gamma$ should wet the surface of $\mathrm{Ni}$, while Ni will agglomerate on $\mathrm{Ti}$ resulting in a rougher Ni-on-Ti interface. The obtained interface rms roughnesses $(\sigma)$ are given in Table I. In order to confirm the role of surfactant in reducing the roughness of Ni-on-Ti interface, we also prepared a $\mathrm{Ni}$ layer and a $\mathrm{Ti} / \mathrm{Ni}$ bilayer on a $\mathrm{Ti}$ buffer layer. In these samples $[\operatorname{Ti}(10 \mathrm{~nm}) / x / \mathrm{Ni}(4 \mathrm{~nm})]$ and $[\operatorname{Ti}(10 \mathrm{~nm}) / x / \operatorname{Ti}(6 \mathrm{~nm}) /$ $\mathrm{Ni}(4 \mathrm{~nm})]$ ( $x=0$ or Ag surfactant) XRR measurements reveal that the roughness of Ni-on-Ti interface decreases significantly with addition of $0.2 \mathrm{~nm} \mathrm{Ag}$ surfactant.

A decrease in the interface roughness results in an increased $\mathcal{R}$. By adding $\mathrm{Ag}$ either on the buffer Ti layer or at each Ti layer, the SLD profile becomes symmetric and the overall roughness decreases. Such decrease in the surface roughness of samples prepared using Ag surfactant was also confirmed by atomic force microscopy measurements (not shown). In the case of samples prepared at $E_{\mathrm{Ar}^{+}}=1.00 \mathrm{keV}$ the results are markedly different as $\mathcal{R}$ only increases marginally when the surfactant was used. The SLD depth profiles are also broader with appreciably higher roughnesses. Still, the roughness of Ni-on-Ti interface of $\approx 1 \mathrm{~nm}$ is higher as compared to $\approx 0.6 \mathrm{~nm}$ for Ti-on-Ni interface. Symmetric and smooth interfaces obtained using $\mathrm{Ag}$ surfactant at $E_{\mathrm{Ar}_{\mathrm{r}}^{+}}$ $=0.75 \mathrm{keV}$ are indicative of layer-by-layer type growth ${ }^{18}$ whereas the growth of Ti/Ni multilayers at $E_{\mathrm{Ar}^{+}}=1.00 \mathrm{keV}$ seems to remain unaffected with addition of $\mathrm{Ag}$ surfactant.

The observed results can be understood in terms of energy of the sputtered atoms (or adatoms, $E_{\text {ad }}$ ). Although $E_{\text {ad }}$ is difficult to calculate precisely, the mean values can be estimated using stopping and range of ions in matter (SRIM) calculations. ${ }^{24,25}$ The calculated values of $E_{\text {ad }}$ (eV/atom) at $E_{\mathrm{Ar}^{+}}=0.75 \mathrm{keV}$ for $\mathrm{Ni}$, Ti, and $\mathrm{Ag}$ are $22.6 \pm 0.5,25.3 \pm 2$, and $20.7 \pm 0.5$, respectively whereas at $E_{\mathrm{Ar}^{+}}=1.00 \mathrm{keV}$ they increase to $23 \pm 0.5,33.3 \pm 2$, and $22.2 \pm 0.5$ for $\mathrm{Ni}$, Ti, and $\mathrm{Ag}$, respectively. As can be seen while the $E_{\text {ad }}$ for $\mathrm{Ni}$ and $\mathrm{Ag}$ increases marginally with an increase in incident ion energy, 
for $\mathrm{Ti}$ it increases appreciably. This may results in an increased mobility of the $\mathrm{Ti}$ atoms during condensation at the substrate. As supported by the XRD and TEM measurements, a larger grain size and sharper Ti peaks for the sample prepared at higher $\mathrm{Ar}^{+}$ion energy may be due to the increased mobility of the Ti adatoms. This situation may lead to an enhanced intermixing at the interfaces and the surfactant atoms may migrate in the host Ti layer and therefore will not be able to float-off to the surface. Therefore, the addition of surfactant atoms will have no or only marginal effect on interface asymmetry as observed in this case. This hypotheses is supported by the absence of the top Ag layer for sample $\mathrm{C}$ in this case.

In conclusion, we observe smooth and symmetric interfaces, indicative of layer-by-layer type growth, in Ti/Ni multilayers prepared using $\mathrm{Ag}$ as surfactant at a deposition energy $E_{\mathrm{Ar}^{+}}=0.75 \mathrm{keV}$. However, when this energy is increased at $E_{\mathrm{Ar}^{+}}=1.00 \mathrm{keV}$ there is only a marginal effect due to the increased mobility of $\mathrm{Ti}$ atoms. Therefore, the energy of the adatoms play a significant role in the mechanism of the surfactant mediated growth of Ti/Ni multilayers.

We acknowledge DST, Government of India for providing financial support to carry out NR experiments under its scheme "Utilization of International Synchrotron Radiation and Neutron Scattering facilities." A part of this work was performed under the Indo-Swiss Joint Research Programme with Grant No. INT/SWISS/JUAF(9)/2009. Thanks to A. Wildes for the help provided in the NR measurements at ILL, N. P. Lalla in TEM measurements, and S. Potdar in sample preparation. A part of this work is based on experiments performed at the Swiss spallation neutron source SINQ, Paul Scherrer Institute, Villigen, Switzerland.

${ }^{1}$ H. Ju and B. J. Heuser, Appl. Phys. Lett. 90, 073113 (2007).

${ }^{2}$ J. Padiyath, J. Stahn, M. Horisberger, and P. Böni, Appl. Phys. Lett. 89, 113123 (2006)
${ }^{3}$ M. S. Kumar, P. Böni, and D. Clemens, J. Appl. Phys. 84, 6940 (1998).

${ }^{4}$ H.-C. Mertins, F. Schäfers, H. Grimmer, D. Clemens, P. Böni, and M. Horisberger, Appl. Opt. 37, 1873 (1998).

${ }^{5}$ J. Chaudhuri, S. Alyan, and A. Jankowski, Thin Solid Films 239, 79 (1994)

${ }^{6}$ H. Takenaka, H. Ito, K. Nagai, Y. Muramatsu, E. Gullikson, and R. Perera, Nucl. Instrum. Methods Phys. Res. A 467-468, 341 (2001).

${ }^{7}$ Q. Su, Y. Zheng, and M. Wuttig, Appl. Phys. Lett. 73, 750 (1998).

${ }^{8}$ M. A. Hollanders, B. J. Thijsse, and E. J. Mittemeijer, Phys. Rev. B 42 5481 (1990).

${ }^{9}$ R. Gupta, M. Gupta, S. Kulkarni, S. Kharrazi, A. Gupta, and S. Chaudhari, Thin Solid Films 515, 2213 (2006).

${ }^{10}$ S. Singh, S. Basu, P. Bhatt, and A. K. Poswal, Phys. Rev. B 79, 195435 (2009).

${ }^{11}$ M. Mâcaza, Z. Jiang, F. Samuel, B. Farnoux, and B. Vidal, J. Appl. Crystallogr. 25, 789 (1992).

${ }^{12}$ M. Hino, H. Sunohara, Y. Yoshimura, R. Maruyama, S. Tasaki, H. Yoshino, and Y. Kawabata, Nucl. Instrum. Methods Phys. Res. A 529, 54 (2004).

${ }^{13}$ M. Ay, C. Schanzer, M. Wolff, and J. Stahn, Nucl. Instrum. Methods Phys. Res. A 562, 389 (2006).

${ }^{14}$ W. Tyson and W. Miller, Surf. Sci. 62, 267 (1977).

${ }^{15}$ S. M. Foiles, M. I. Baskes, and M. S. Daw, Phys. Rev. B 33, 7983 (1986).

${ }^{16}$ M. S. Kumar, P. Böni, S. Tixier, and D. Clemens, Physica B 241-243, 95 (1997).

${ }^{17}$ M. Copel, M. C. Reuter, E. Kaxiras, and R. M. Tromp, Phys. Rev. Lett. 63, 632 (1989).

${ }^{18}$ A.-L. Barabási, Phys. Rev. Lett. 70, 4102 (1993).

${ }^{19}$ T. Schmidt, R. Kröger, T. Clausen, J. Falta, A. Janzen, M. Kammler, P. Kury, P. Zahl, and M. H. von Hoegen, Appl. Phys. Lett. 86, 111910 (2005).

${ }^{20}$ C. Tölkes, R. Struck, R. David, P. Zeppenfeld, and G. Comsa, Phys. Rev. Lett. 80, 2877 (1998)

${ }^{21}$ M. Gupta, A. Gupta, S. M. Amir, J. Stahn, and J. Zegenhagen, J. Phys.: Conf. Ser. 211, 012020 (2010).

${ }^{22}$ M. Gupta, T. Gutberlet, J. Stahn, P. Keller, and D. Clemens, Pramana, J. Phys. 63, 57 (2004).

${ }^{23}$ C. Braun, PARRATT32-the reflectivity tool, HMI Berlin, 1997.

${ }^{24}$ J. P. Biersack and W. Eckstein, Appl. Phys. A: Mater. Sci. Process. 34, 73 (1984).

${ }^{25}$ J. F. Ziegler, J. P. Biersack, and M. D. Ziegler, SRIM 2010, The Stopping and Range of Ions in Matter, online at: www.srim.org. 